\title{
Coherent synthesis of ultra-broadband optical parametric amplifiers
}

\author{
C. Manzoni, ${ }^{1, *}$ S.-W. Huang, ${ }^{2}$ G. Cirmi, ${ }^{2,3}$ P. Farinello, ${ }^{1}$ J. Moses, ${ }^{2}$ F. X. Kärtner, ${ }^{2,3}$ and G. Cerullo ${ }^{1}$ \\ ${ }^{1}$ IFN-CNR, Dipartimento di Fisica, Politecnico di Milano, Piazza Leonardo da Vinci 32, I-20133 Milan, Italy \\ ${ }^{2}$ Department of Electrical Engineering and Computer Science and Research Laboratory of Electronics, \\ Massachusetts Institute of Technology, Cambridge, Massachusetts 02139, USA \\ ${ }^{3}$ Center for Free-Electron Laser Science, DESY and Department of Physics, University of Hamburg, \\ Notkestrasse 85, D-22607 Hamburg, Germany \\ *Corresponding author: cristian.manzoni@polimi.it
}

Received January 25, 2012; revised March 14, 2012; accepted March 18, 2012; posted March 20, 2012 (Doc. ID 162019); published May 21, 2012

\begin{abstract}
We report on coherent synthesis of two ultra-broadband optical parametric amplifiers, each compressed by chirped mirror pairs, resulting in almost-octave-spanning (520-1000 nm) spectra supporting nearly single-cycle sub-4 fs pulse duration. Synthesized pulse timing is locked to less than 30 as by a balanced optical cross-correlator. The synthesized pulse is characterized by two-dimensional spectral interferometry and has a 3.8 fs duration. (C) 2012 Optical Society of America
\end{abstract}

OCIS codes: $190.4970,190.2620,320.7110$.

The generation of single-cycle, carrier-envelope phase (CEP)-stable light pulses is one of the frontiers of ultrafast optics, promising to break tremendous new ground in the control of strong-field processes such as highharmonic and attosecond pulse generation [1]. The two key ingredients for single-cycle pulse generation are ultrabroad bandwidths, exceeding 1 octave, and accurate control of the spectral phase, so as to obtain nearly transform-limited (TL) pulse widths. These requirements are very challenging to satisfy with a single laser source or optical amplifier, and thus, intense research activity has been devoted to the coherent synthesis of pulses generated from separate femtosecond laser sources [2,3]. Coherent addition of two pulse trains derived from the same fiber laser and spectrally broadened by self-phasemodulation (SPM) in an optical fiber resulted in the first demonstration of an isolated single-cycle optical pulse [4], although with low (nanojoule) energy. SPM in a hollow-core fiber, followed by dispersion management of different portions of the ultra-broadband spectrum and coherent superposition of the compressed subpulses, has recently allowed the generation of subcycle light pulses with energies of hundreds of microjoules []. However, because of the high pressures used in the hollow-fiber compressor, such pulses show strongly modulated spectra and further scaling is limited by the energy-handling capabilities of the fiber.

Coherent combination of ultra-broadband optical parametric amplifiers (OPAs) or optical parametric chirpedpulse amplifiers (OPCPAs) is a promising alternative route to high-energy single-cycle pulse synthesis, with the advantages of smooth spectra and straightforward energy scaling capabilities. A first milestone in this direction was the recent demonstration of the synthesis of two OPCPAs, at $800 \mathrm{~nm}$ and $2 \mu \mathrm{m}$, which enabled subcycle waveform shaping over a spectrum spanning 2 octaves [6]. Here we demonstrate coherent synthesis of two spectrally adjacent ultra-broadband OPAs pumped by a single Ti:sapphire laser and seeded by the same white-light continuum (WLC), resulting in a nearly octave-spanning, gap-free spectrum.
OPAs are powerful tools for the generation of tunable, few-optical-cycle light pulses [7]]. Broadband gain in an OPA is achieved when the group velocities of signal and idler are matched [8]; this condition is satisfied either in the case of a degenerate OPA (DOPA) with type I phase matching or in the noncollinear OPA (NOPA), where the idler group velocity projected along the signal propagation direction is matched to the signal group velocity. Using these concepts, a variety of broadband OPA schemes, pumped by either the fundamental frequency (FF) or the second harmonic (SH) of Ti:sapphire, have been demonstrated. In particular, sub-10 fs visible pulses (500-700 $\mathrm{nm}$ ) are routinely generated by the SH-pumped NOPA [9], and sub-7 fs pulses have been obtained in the $650-950 \mathrm{~nm}$ spectral region by the SH-pumped DOPA [10]. Here we demonstrate coherent synthesis of the SHpumped NOPA and DOPA, resulting in ultra-broadband (520-1000 nm) spectra supporting nearly single-cycle sub-4 fs pulses. After compressing the individual subpulses with chirped mirrors, the combined pulse has a nearly TL 3.8 fs duration (1.6 cycles at $700 \mathrm{~nm}$ ).

The experimental setup for coherent synthesis of OPAs, summarized in the block diagram of Fig. 1(a), consists of (i) a WLC seed generation stage, (ii) two broadband OPAs seeded by distinct portions of the WLC, and (iii) coherent combination of their outputs. The system is powered by an amplified Ti:sapphire laser, providing $550 \mu \mathrm{J}, 130 \mathrm{fs}$ pulses at $800 \mathrm{~nm}$ and $1 \mathrm{kHz}$. Since the WLC presents highly structured intensity and spectral phase profiles around the driving pulse wavelength, it is not possible to use the WLC directly generated by the FF to seed the DOPA. Therefore, an additional stage is necessary to shift the WLC driving wavelength to the infrared (IR), thus generating a well-behaved WLC around $800 \mathrm{~nm}$ [10]. This twostage IR OPA, using type II $\beta$-barium borate (BBO) crystals, is pumped by the $\mathrm{FF}$ of Ti:sapphire $(250 \mu \mathrm{J})$ and seeded by the supercontinuum generated in a sapphire plate [11]. Both the signal and the CEP-stable idler [12] from the IR OPA can be used to generate the WLC seed [13]. For this experiment, we use the signal at $1.3 \mu \mathrm{m}$ to generate the seed in a 3-mm thick sapphire plate. 


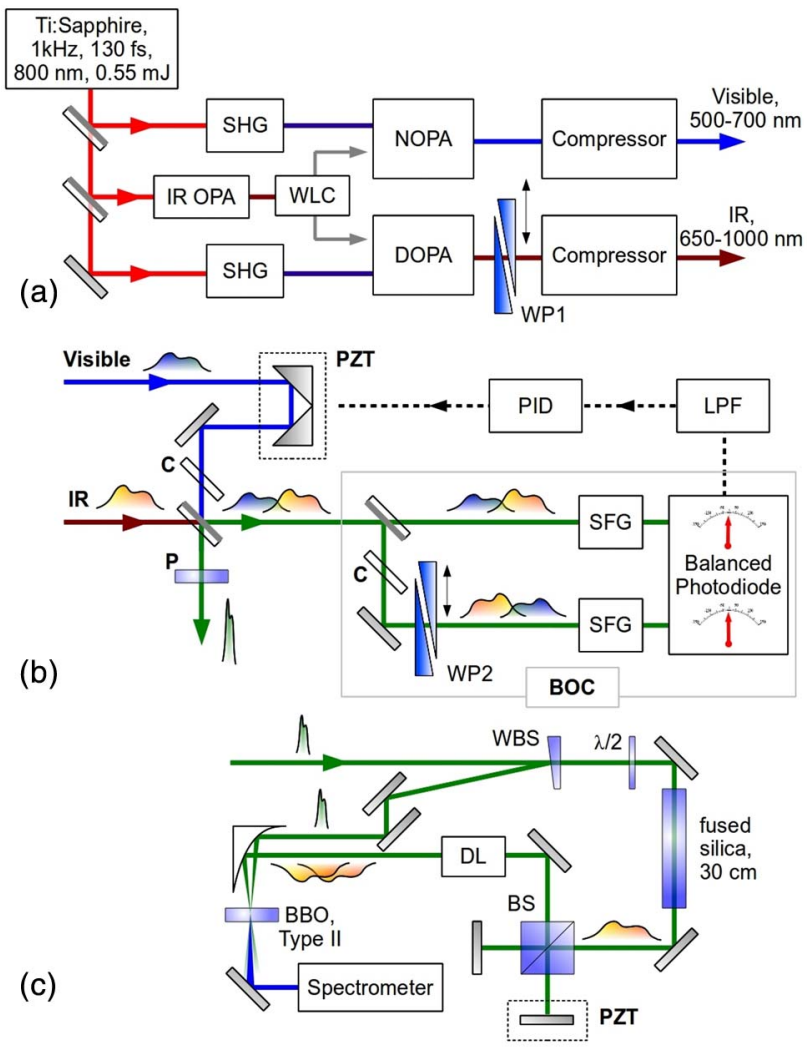

Fig. 1. (Color online) (a) Setup for separate amplification, compression, and synthesis of visible and infrared pulses. SHG: second-harmonic generation; WP1: fused silica wedge plates for the tuning of the visible-IR relative phase. (b) Schematic of the BOC. PZT: piezoelectric actuator; $\mathrm{WP} 2: \mathrm{CaF}_{2}$ wedge plates for delay tuning; $\mathrm{P}$ : 2-mm thick $\mathrm{CaF}_{2}$ plate; $\mathrm{C}$ : compensation plates; LPF: electronic low-pass filter; SFG: sum-frequency generation. (c) 2DSI schematic. BS: beam splitter; WBS: wedge beam splitter; DL: delay line.

The short-wavelength portion of this seed ranges from 500 to $1000 \mathrm{~nm}$ (Fig. 2(a), solid line) and covers the gain range of the visible NOPA and SH-pumped DOPA (Fig 2(a), dashed lines). We split the seed with a neutral-

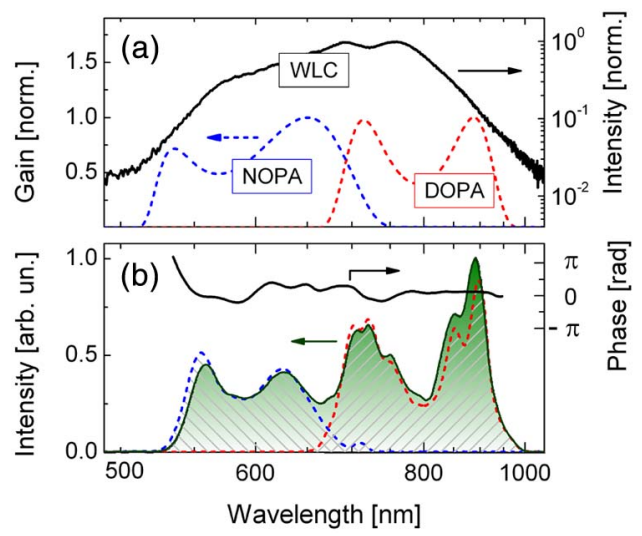

Fig. 2. (Color online) (a) Spectrum of the gap-free WLC (solid line, log scale) generated in the sapphire plate compared with the calculated gain of the visible and IR OPAs (dashed lines, linear scale). (b) Spectra of the individual OPA pulses (dashed lines) and of the synthesized pulses. The spectral phase (solid black line) is deduced from the 2DSI measurement. density ultrathin beam splitter and amplified the two replicas with the visible NOPA and the DOPA. Both OPAs were pumped by the SH from the remaining $300-\mu \mathrm{J} \mathrm{FF}$ pulses; we used thick SH crystals to narrow the bandwidth of the SH pulse, facilitating its temporal overlap with the chirped WLC. The visible NOPA employed a $1 \mathrm{~mm}$-thick type I BBO crystal cut at $\theta=32^{\circ}$ and an external pump signal angle of $\approx 6^{\circ}$, while the DOPA used a $1 \mathrm{~mm}$-thick BBO with $\theta=29^{\circ}$ and a nearly collinear interaction; each OPA generates pulses with $1-2 \mu \mathrm{J}$ energy. To ensure spatial overlap of the collimated beams, we used the same focal lengths and propagated the beams for the same distances in the two OPAs. We compressed the two OPA outputs by broadband visible [14] and IR [15] chirped mirrors, which provided pulse durations close to the TL values ( $\approx 7$ fs for both OPAs). We then synchronized the two amplified pulses with a delay line equipped with a piezoelectric actuator (PZT) and collinearly combined them with an ultrathin metallic beam splitter with spectrally flat 50:50 splitting ratio. A gapfree spectrum arising from the combination of the two pulses is shown on a linear scale in Fig. 2(b); it ranges from 520 to $1000 \mathrm{~nm}$ with very good spectral uniformity and supports a TL 3.65 fs pulse duration.

The last step of the pulse synthesis is the coherent combination of the two pulses, which calls for careful control of their relative delay and CEP. The relative CEP can be finely tuned by wedge pair WP1 (Fig. 1(a)). We detected the relative delay between the two pulses with the balanced optical cross-correlator (BOC) [16] sketched in Fig 1(b), where two replicas of the synthesized pulse with opposite chirp are cross-correlated in 1 mm-thick BBO crystals, phase-matched for sumfrequency generation of $600 \mathrm{~nm}$ light from the NOPA and $800 \mathrm{~nm}$ light from the DOPA. Thanks to balanced detection, the BOC allows attosecond-precision relative timing measurement. We sent the low-pass $(<30 \mathrm{~Hz})$ filtered BOC signal into a feedback loop based on a proportional-integral-derivative controller driving the PZT. The feedback locks the relative delay between the pulses, tuned by WP2, with fluctuations below 30 as (rms) over a measurement time of $10 \mathrm{~min}$, corresponding to less than $1 / 80$ optical cycle (Fig. 3). Temporal characterization of the synthesized pulse was performed with twodimensional spectral shearing interferometry (2DSI) [17], which upconverts the pulse to be measured with two strongly chirped and time-delayed replicas, encoding the frequency-dependent group delay (GD) in the interference between the two temporally overlapped upconverted pulses; thanks to its ultrabroad acceptance bandwidth and the avoidance of delay calibration, it is

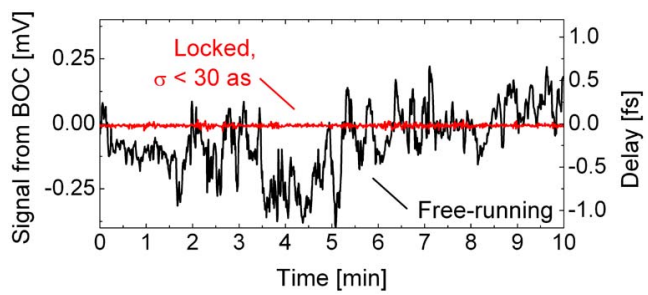

Fig. 3. (Color online) Relative pulse delay deduced from the BOC signal, measured in free-running and in closed feedback loop. 

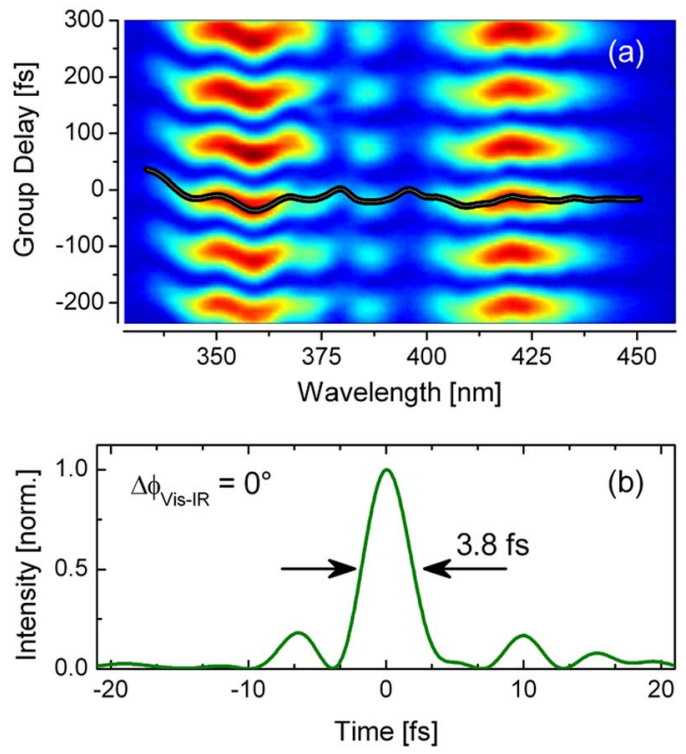

Fig. 4. (Color online) (a) 2DSI map and retrieved group delay; the signal arises from sum-frequency generation of the whole pulse with components at $900 \mathrm{~nm}$. (b) Intensity profile of the reconstructed pulse, assuming a continuous phase between visible and infrared fields.

suited for the characterization of octave-spanning pulses. Our implementation of 2DSI is shown in Fig. 1(c); we chirp the IR portion of the spectrum, and the $40 \mu \mathrm{m}$ type II BBO upconversion crystal guarantees a broad acceptance bandwidth along the ordinary axis.

Figure 4(a) shows a 2DSI map of the synthesized spectrum and the retrieved frequency-dependent GD, which is substantially flat over the whole pulse bandwidth; the residual GD oscillations in the visible spectral range are due to the large number of bounces on the chirped mirrors. The retrieved spectral phase, reported in Fig. 2(b), was applied to the measured spectral amplitude, giving the pulse shown in Fig. 4(b). The FWHM of its intensity profile is $3.8 \mathrm{fs}$, indicating that the pulse is compressed to within $4 \%$ of the TL duration. The good compression and the gap-free, smooth synthesized spectrum concentrate $70 \%-80 \%$ of the pulse energy in the main lobe; in addition, the oscillation of the electric field in the sidelobes is small enough to have a negligible contribution to strong-field processes, such as CEP-sensitive field emission [18].

In conclusion, we have demonstrated coherent synthesis of pulses from two ultra-broadband OPAs, pumped by the SH of Ti:sapphire; the combined spectrum extends from 520 to $1000 \mathrm{~nm}$, corresponding to $3.65 \mathrm{fs}$ TL pulses. We locked the relative timing of the two OPAs to less than 30 as, using a feedback loop based on a BOC. The synthesized pulse duration is $3.8 \mathrm{fs}$, close to the TL value; by seeding the OPAs with CEP-stable light, the scheme allows the generation of nearly single-cycle pulses with fully controlled electric field profile. This is a low-power proof-of-principle experiment demonstrating that OPAs are good alternatives to the more complex OPCPA schemes for the synthesis of ultra-broadband pulses.
Scaling of the energy of both OPAs to the millijoule level by addition of power amplification stages should be straightforward [19]. Furthermore, the extension of wavelength multiplexing by the coherent addition of other ultra-broadband OPAs [20] will allow the generation of high-quality subcycle pulses.

This work was supported by the Air Force Office of Scientific Research (grants FA8655-09-1-3101 and FA9550-10-1-0471) and by the Progetto Roberto Rocca.

\section{References}

1. E. Goulielmakis, M. Schultze, M. Hofstetter, V. S. Yakovlev, J. Gagnon, M. Uiberacker, A. L. Aquila, E. M. Gullikson, D. T. Attwood, R. Kienberger, F. Krausz, and U. Kleineberg, Science 320, 1614 (2008).

2. K. Shelton, L.-S. Ma, H. C. Kapteyn, M. M. Murnane, J. L. Hall, and J. Ye, Science 293, 1286 (2001).

3. J. Cox, A. Sell, A. Leitenstorfer, and F. X. Kärtner, "Coherent synthesis of independent, ultrafast lasers for single-cycle pulse generation," presented at Ultrafast Optics 2011, Monterey, CA, 26-30 Sept. 2011.

4. G. Krauss, S. Lohss, T. Hanke, A. Sell, S. Eggert, R. Huber, and A. Leitenstorfer, Nature Photonics 4, 33 (2010).

5. A. Wirth, M. T. Hassan, I. Grguraš, J. Gagnon, A. Moulet, T. T. Luu, S. Pabst, R. Santra, Z. A. Alahmed, A. M. Azzeer, V. S. Yakovlev, V. Pervak, F. Krausz, and E. Goulielmakis, Science 334, 195 (2011).

6. S.-W. Huang, G. Cirmi, J. Moses, K.-H. Hong, S. Bhardwaj, J. R. Birge, L.-J. Chen, E. Li, B. J. Eggleton, G. Cerullo, and F. X. Kärtner, Nature Photonics 5, 475 (2011).

7. G. Cerullo and S. De Silvestri, Rev. Sci. Instrum. 74, 1 (2003).

8. D. Brida, C. Manzoni, G. Cirmi, M. Marangoni, S. Bonora, P. Villoresi, S. De Silvestri, and G. Cerullo, J. Opt. 12, 013001 (2010).

9. A. Baltuška, T. Fuji, and T. Kobayashi, Opt. Lett. 27, 306 (2002).

10. M. Siddiqui, G. Cirmi, D. Brida, F. X. Kärtner, and G. Cerullo, Opt. Lett. 34, 3592 (2009).

11. R. A. Kaindl, M. Wurm, K. Reimann, P. Hamm, A. M. Weiner, and M. Woerner, J. Opt. Soc. Am. B 17, 2086 (2000).

12. A. Baltuška, T. Fuji, and T. Kobayashi, Phys. Rev. Lett. 88, 133901 (2002).

13. G. Cirmi, C. Manzoni, D. Brida, S. De Silvestri, and G. Cerullo, J. Opt. Soc. Am. B 25, B62 (2008).

14. M. Zavelani-Rossi, G. Cerullo, S. De Silvestri, L. Gallmann, N. Matuschek, G. Steinmeyer, U. Keller, G. Angelow, V. Scheuer, and T. Tschudi, Opt. Lett. 26, 1155 (2001).

15. T. R. Schibli, O. Kuzucu, J. Kim, E. P. Ippen, J. G. Fujimoto, F. X. Kärtner, V. Scheuer, and G. Angelow, IEEE J. Sel. Top. Quantum Electron. 9, 990 (2003).

16. T. R. Schibli, J. Kim, O. Kuzucu, J. T. Gopinath, S. N. Tandon, G. S. Petrich, L. A. Kolodziejski, J. G. Fujimoto, E. P. Ippen, and F. X. Kärtner, Opt. Lett. 28, 947 (2003).

17. J. R. Birge, H. M. Crespo, and F. X. Kärtner, J. Opt. Soc. Am. B 27, 1165 (2010).

18. M. Krüger, M. Schenk, and P. Hommelhoff, Nature 475, 78 (2011).

19. P. Tzankov, J. Zheng, M. Mero, D. Polli, C. Manzoni, and G. Cerullo, Opt. Lett. 31, 3629 (2006).

20. D. Brida, G. Cirmi, C. Manzoni, S. Bonora, P. Villoresi, S. De Silvestri, and G. Cerullo, Opt. Lett. 33, 741 (2008). 